\title{
Plasma Simulation Data Through the Hololens
}

\author{
Greg Foss \\ Texas Advanced Computing Center \\ The University of Texas at Austin \\ Austin, TX, USA \\ foss@tacc.utexas.edu
}

\author{
Andrew Solis \\ Texas Advanced Computing Center \\ The University of Texas at Austin \\ Austin, TX, USA \\ asolis@tacc.utexas.edu
}

\author{
Sarang Bhadsavle \\ Texas Advanced Computing Center \\ The University of Texas at Austin \\ Austin, TX, USA \\ sbhadsavle@tacc.utexas.edu
}

\author{
Wendell Horton \\ Institute for Fusion Studies \\ The University of Texas at Austin \\ Austin, TX, USA \\ horton@physics.utexas.edu
}

\author{
Lee Leonard \\ Institute for Fusion Studies \\ The University of Texas at Austin \\ Austin, TX, USA \\ cloud@utexas.edu
}

\begin{abstract}
The Texas Advanced Computing Center (TACC) demonstrates scientific visualization with Microsoft's Hololens [1]. In a mixed reality (MR) environment this tool allows the scientist to see their data geometry as a holographic image with the ability to freely walk around and view the model as if it were a free-floating object.

We presented our first complete prototype at the SC17 conference [2] in Denver. This shows a looping animation representing 300 timesteps of data from a mirror machine magnetic confinement simulation, and includes a short narration voice-over. The data is a rotating cylinder of plasma density that gradually loses stability resulting in collapse. Physicist Wendell Horton, from the Institute for Fusion Studies at the University of Texas at Austin, uses TACC's Stampede2 supercomputer for his simulations investigating plasma turbulence as applied to research in nuclear fusion and Earth's ionosphere.

Currently we're using ParaView [3] and Unity [4] software to build the visualization content and are experimenting with more elaborate plasma visualizations with planned additional projects.
\end{abstract}

\section{CCS CONCEPTS}

- Human-centered computing $\rightarrow$ Scientific visualization;

\section{KEYWORDS}

plasma simulation, visualization, augmented reality, mixed reality, Hololens, nuclear fusion

\section{ACM Reference Format:}

Greg Foss, Andrew Solis, Sarang Bhadsavle, Wendell Horton, and Lee Leonard. 2018. Plasma Simulation Data Through the Hololens. In PEARC '18: Practice and Experience in Advanced Research Computing, July 22-26, 2018, Pittsburgh, PA, USA. ACM, New York, NY, USA, 2 pages. https://doi. org/10.1145/3219104.3229431

Permission to make digital or hard copies of part or all of this work for personal or classroom use is granted without fee provided that copies are not made or distributed for profit or commercial advantage and that copies bear this notice and the full citation on the first page. Copyrights for third-party components of this work must be honored.

For all other uses, contact the owner/author(s).

PEARC '18, fuly 22-26, 2018, Pittsburgh, PA, USA

(C) 2018 Copyright held by the owner/author(s).

ACM ISBN 978-1-4503-6446-1/18/07.

https://doi.org/10.1145/3219104.3229431

\section{INTRODUCTION: WHY THE HOLOLENS}

Virtual reality (VR) devices, such as the Oculus [5] or HTC VIVE [6], provide a visualization experience as if transporting the user into another world, an environment defined by the data. In this project we didn't want to completely move the user from one reality to another, but instead allow them to focus on the structure formed by their data, visualized like an individual object. VR can be effective if the user needs to be inside their data, for example if Dr. Horton wanted to study and experience plasma at close range.

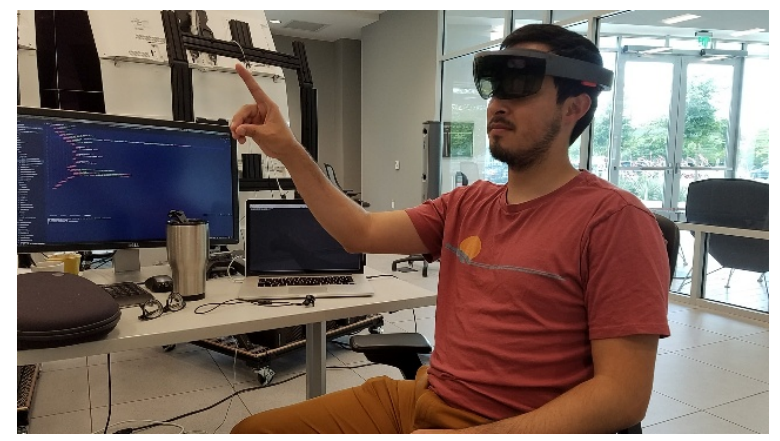

Figure 1: Co-author A.Solis selecting from a menu displayed in the Hololens

\section{PROCESS}

With the SC17 conference imminent we wanted to get a quick proofof-concept. We used ParaView and Horton's plasma turbulence simulation data to create an isosurface model of plasma density from a value that appears as a spinning cylinder that gradually loses stability and suddenly collapses. In order to view this with the Hololens we used a Filmbox (FBX)[7] exporter ParaView plugin [8] to output geometry that could be read into Unity. In Unity we created a scene from each timestep, added color and lighting, and read these into the Hololens stored for playback. Though limited by the Hololens 64 GB storage limit, this was a quick way to get the experiment working- using familiar tools.

A compromise between the Hololens space limit and a satisfying visualization required some trial and error. We used ParaView's decimate filter to reduce triangle number and file size, yet maintain 
geometry that still closely matched the original. We also used every third model- equivalent to playing an animation "on 3's." The project was completed with a script displaying the scenes, looping the animation until the user stopped the application (or the device's charge ran out). An added voice-over introduced Horton's research.

We're currently looking for ways to stream modelled data to the Hololens to get around its storage limitation. One possibility might be with Microsoft's DirectX [9] application programming interface.


Figure 2: Co-author W. Horton checking out his data in the Hololens

\section{SCIENCE}

The visualizations show how the relatively simple high-temperature mirror plasma, with intrinsic rotation from the faster axial lossrate of electrons than ions, evolves into complex rotational modes. Though complicated engineering methods were developed in attempts to control these rotational instabilities, the modes still persisted and eventually, in less than one second, would develop complex structures and terminate the confinement. The visualizations provide a $3 \mathrm{D}$ picture of these complex nonlinear dynamics that result from nonlinear growth of the initial relatively simple linear eigenmodes of the mirror machine. The results also apply approximately to the mirror modes that develop in the Earth's inner magnetic sphere which is a nearly collisionless mirror controlled plasma. In general the 3D visualizations show how complex and multi-scaled the nonlinear dynamics have become.

Investigation of jet structures with vortical motion is a fundamental key for describing solar tornadoes as well as astrophysical jets. Investigation of jets as collective motion is key for understanding the energy transfer in turbulent magnetized plasma, although the real system is obviously more complicated then this axially symmetrical model. As in the Burgers vortex, the inward, radial flow tends to concentrate vorticity in a narrow column around the symmetry axis. At the same time, the vertical flow tends to spread the vorticity and the stationary vortex arises when the two effects are balanced. The developed model may be used to illustrate the vortex stretching mechanism and may describe such flows as tornados, where the vorticity is provided by continuous convection-driven vortex stretching. Please refer to publications ITER Physics [Horton-2015] and Turbulent Transport in Magnetized Plasmas [Horton-2012] for a thorough explanation of Horton's research.

\section{CONCLUSION}

One insight gained from working with Dr. Horton and MR was usability. Dr. Horton is not an MR enthusiast or gamer, nor directly involved in visualization development so watching him use the Hololens demonstrated practical considerations like his comfort both wearing the device and also using it. It doesn't matter how forward-thinking a technology is if it breaches the participant's patience limit. It's also important to consider the Hololens's notlarge viewing area and how appropriate that size is relative to the data's complexity. Once he learned how to use his fingers for control, where to look for the animation, and how to start it, our cherished scientist guinea pig stood up, started walking back and forth muttering about waves, and clearly enjoyed this new way to see his data (note posture in Figure 2).

Our experiment with the Hololens does not show more data than with conventional visualization animation and images, but it does allow its user viewpoints with the same immediacy as walking around a 3D object, turning their head as they please to observe. We're currently experimenting with a plasma model that has structures in multiple areas that overlap. Conventional slices, cutaways, fly-throughs and transparency allow visibility, but perhaps instead seeing the data intact with that immediate visual feedback will reveal new visualization possibilities. Dr. Horton is impressed with the experience of visualizing his plasma in an AR environment, but it's a new approach for him and he has to consider how this technology could be used to aid his discovery process.

\section{ACKNOWLEDGMENTS}

W. Horton's research is funded in part by US Department of Energy grant DE-FG02-04ER54742.

Thanks to fellow TACC staff, especially Anne Bowen and others of the visualization groups, for technical support and helpful advice, and to Wendell Horton for his energizing, and entertaining, enthusiasm for scientific visualization.

\section{REFERENCES}

[1] https://www.microsoft.com/en-us/hololens

[2] https://sc17.supercomputing.org/

[3] https://www.paraview.org/

[4] https://unity3d.com/

[5] http://www.oculus.com

[6] https://www.vive.com/us/

[7] https://www.autodesk.com/products/fbx/overview

[8] https://github.com/ufz-vislab/VtkFbxConverter

[9] https://developer.nvidia.com/directx

[Horton-2015] Horton, W., Benkadda, S., 2015, ITER Physics, World Scientific

[Horton-2012] Horton, W., 2012, Turbulent Transport in Magnetized Plasmas, 2nd Edition, World Scientific

Received May 2018 